\title{
Special issue on PODC 2009
}

\author{
Lorenzo Alvisi
}

Published online: 30 August 2011

(C) Springer-Verlag 2011

This special issue of Distributed Computing is based on four papers that originally appeared, in preliminary and abbreviated form, in the Proceedings of the 28th ACM Symposium on the Principles of Distributed Computing (PODC 2009), held in Calgary, Canada, on August 10-12. The papers were chosen by the Program Committee from the 27 full-length papers presented at the Symposium and are fine examples of the quality and diversity of the research presented at PODC.

In the first paper, Randomized Mutual Exclusion with Sub-Logarithmic RMR-Complexity, Hendler and Woelfel shed new light on the fundamental mutual exclusion problem, showing that randomized mutual exclusion algorithms that use only reads and writes can overcome the logarithmic lower bound shown by Attiya, Hendler, and Woelfel for deterministic algorithms, and achieve sub-logarithmic complexity when measured in terms of remote memory references (RMRs).

The second paper, Load Balancing Without Regret in the Bulletin Board Model presents an excellent example of the growing interest of the PODC community in algorithmic game theory. Kleinberg, Piliouras, and Tardos study the performance of a simple, well-known, no-regret multiplicativeweights algorithm for a class of load balancing games in a model where players can query the current state of the system but cannot reliably predict the effect of their actions on it. Using non-standard analysis, they show that the makespan (i.e. the maximum load over all machines) achieved by this simple algorithm deviates in any step by at most $O(\log n)$ from the optimal makespan, a result that is exponentially

L. Alvisi $(\varangle)$

Department of Computer Science,

The University of Texas at Austin, Austin, TX, USA

e-mail: lorenzo@cs.utexas.edu better than the known lower bounds for generic no-regret algorithms.

Parsimonious Flooding in Dynamic Graphs, by Baumann, Crescenzi, and Fraigniaud studies an interesting variant of the flooding protocol over a class of evolving graphs generated through an edge-Markovian process. In this variant, every node sends the source message only for a bounded number of time after its reception. At the core of the paper is an insightful lemma that provides a reduction from a timebased edge-Markovian series of graphs to a single random graph. With this reduction, the analysis of the number $k$ of rounds needed for the flooding to succeed, as well as the flooding time, reduce elegantly to questions of connectivity and diameter of the random graph.

Finally, Distributed Algorithms for Covering, Packing and Maximum Weighted Matching advances an important line of research that, in recent years, has applied LP-based methods to develop efficient distributed algorithms for many basic graph-theoretic problems. Koufogiannakis and Young's results are elegant and powerful and solve basic open problems in distributed/parallel computation, such as finding an efficient 2-approximation for weighted vertex cover.

After being reviewed, in preliminary form, by the Program Committee of PODC 2009, these papers were refereed according to the standard practices of Distributed Computing. Thank you once more to both the authors and the referees, and to Hagit Attiya, who as the journals' Editor in Chief provided her warm support and advice through this process.

Lorenzo Alvisi

Program Chair, PODC 2009

Guest Editor 